\title{
Two-dimensional spectra of electron collisions with acrylonitrile and methacrylonitrile reveal nuclear dynamics
}

\author{
K. Regeta ${ }^{a)}$ and M. Allan ${ }^{\text {b) }}$ \\ Department of Chemistry, University of Fribourg, Chemin du Musée 9, CH-1700 Fribourg, Switzerland
}

\begin{abstract}
Detailed experimental information on the motion of a nuclear packet on a complex (resonant) anion potential surface is obtained by measuring 2-dimensional (2D) electron energy loss spectra. The cross section is plotted as a function of incident electron energy, which determines which resonant anion state is populated, i.e., along which normal coordinate the wave packet is launched, and of the electron energy loss, which reveals into which final states each specific resonant state decays. The 2D spectra are presented for acrylonitrile and methacrylonitrile, at the incident energy range $0.095-1.0 \mathrm{eV}$, where the incoming electron is temporarily captured in the lowest $\pi^{*}$ orbital. The 2D spectra reveal selectivity patterns with respect to which vibrations are excited in the attachment and de-excited in the detachment. Further insight is gained by recording 1D spectra measured along horizontal, vertical, and diagonal cuts of the 2D spectrum. The methyl group in methacrylonitrile increases the resonance width 7 times. This converts the sharp resonances of acrylonitrile into boomerang structures but preserves the essence of the selectivity patterns. Selectivity of vibrational excitation by higher-lying shape resonances up to $8 \mathrm{eV}$ is also reported.
\end{abstract}

\section{INTRODUCTION}

Dissociative electron attachment (DEA) is perhaps the most important category of electron-driven chemistry and has numerous applications in industrial, medical, atmospheric, and interstellar processes, justifying the quest for understanding the motion of the nuclei on the complex potential surface which it involves. A particularity of the motion of a nuclear wave packet on a complex potential surface of a transient anion, which distinguishes it from the case of a neutral molecule encountered in photochemistry, is the competition with fast autodetachment, the rate of which is expressed as a width $\Gamma(R)$ (where $R$ is the nuclear coordinate). The exciting aspect of the detachment is that the departing electrons in a certain sense "report" on the progress of the nuclear motion because the detachment generally leads to a vibrationally excited neutral molecule and the selectivity with respect to which vibrational modes are excited carries information about the path of the nuclear wave packet on the complex potential surface. The present paper elaborates on the information gained in this way.

In our earlier paper, ${ }^{1}$ we pointed out that 2 -dimensional (2D) spectra are a powerful presentation of the wealth of information gained from the detached electrons. The 2D spectra plot the detached electron yield as a function of the incident electron energy, which determines along which vibrational mode the nuclear wave packet is "sent off," and of the electron-energy loss, which indicates into which vibrational mode the molecule finally "landed." This paper reports a more detailed account of the $2 \mathrm{D}$ spectrum of acrylonitrile and extends it to the related molecule methacrylonitrile. Certain aspects are illustrated with "cuts" through the 2D spectra in vertical, horizontal, and

\footnotetext{
a)khrystyna.regeta@unifr.ch

b) michael.allan@unifr.ch
}

diagonal directions. A number of interesting insights into the motion of the nuclei were gained from the spectra.

The usefulness of methacrylonitrile for the present study stems from the larger width of the resonance, which permits investigation of the role of faster autodetachment rate on the nuclear dynamics. The interest in acrylonitrile is augmented by its occurrence in outer space ${ }^{2}$ where it may be exposed to free electrons. Both acrylonitrile and methacrylonitrile are widely used precursors for polymers, including electron sensitive resists.

An important aim of the work is to inspire the development of a unified theory of vibrational excitation (VE) and DEA of polyatomic molecules, along the lines pioneered by Chourou and $\mathrm{Orel}^{3}$ on acetylene, which would, however, calculate not only the final flux of the dissociation products, as was the case in the acetylene work (the calculated flux compared favorably with the absolute DEA cross section ${ }^{4}$ ), but also the spectra of the electrons detached while the nuclear wave packet moves on the complex part of the potential. A comparison with experimental 2D spectra would provide a detailed guidance and a test for the theory in terms of whether it correctly describes the path of the wave packet and $\Gamma(R)$.

The present paper also reports absolute values of the cross sections in acrylonitrile and an overview of higher-lying resonances in both molecules. Citations of earlier work on electron scattering in acrylonitrile, on dipole-bound anions, and on early 2D spectra of electron collisions were given in our previous report. $^{1}$

\section{METHODS}

The measurements were performed using a spectrometer with hemispherical analyzers described earlier. ${ }^{5,6}$ The energy 
of the incident beam was calibrated on the $19.365 \mathrm{eV}{ }^{2} \mathrm{~S}$ resonance in helium ${ }^{7}$ and is accurate to within $\pm 10 \mathrm{meV}$. The instrumental resolution in the energy-loss mode, taken as the width of the elastic peak in acrylonitrile and methacrylonitrile, was $15.5 \mathrm{meV}$. This implies a resolution of $11 \mathrm{meV}$ in the incident beam. Beam current was typically $300 \mathrm{pA}$. The instrumental response function was determined on elastic scattering in helium and all spectra were corrected as described earlier. ${ }^{6,8}$ Absolute values of the cross sections for acrylonitrile were determined by the relative flow technique as described by Nickel et al. ${ }^{9}$ using the theoretical helium elastic cross sections of Nesbet ${ }^{10}$ as a reference. Acrylonitrile "sticks" to the inner surfaces of the gas system, presumably because of its large dipole moment, making the pressure rise slower than would correspond to the flow. The confidence limit is therefore wider than for "nonsticky" compounds and is estimated to be about $\pm 40 \%$. The acrylonitrile and helium pressures in the gas inlet line were typically 0.08 and 0.24 mbars, respectively, during the absolute measurements. The low vapor pressure of methacrylonitrile required the regulating needle valve to be operated wide open and thus did not permit the measurement of absolute values of the cross sections - the requirement that the pressure drop across the regulating valve is large compared to the pressure behind the nozzle was not fulfilled. Only relative cross sections are thus given for methacrylonitrile.

Two-dimensional spectra were obtained by recording a series of 160 electron energy-loss (EEL) spectra with constant incident energies in the range $0.095-0.9 \mathrm{eV}$ and combining them. Each of the 160 spectra was corrected for the response function of the analyzer and the height of the elastic peak was then normalized to the separately measured magnitude of the elastic cross section at each energy. This procedure is slightly different from that used in our earlier work, ${ }^{1}$ where the elastic peak was normalized to 1 for all incident energies. Spectra were recorded at the large scattering angle of $135^{\circ}$ to emphasize resonant processes, which are of interest here, and reduce the extent of direct excitation.

\section{OVERVIEW OF SHAPE RESONANCES}

\section{A. Acrylonitrile}

Burrow et al. ${ }^{15}$ studied shape resonances in acrylonitrile by Electron Transmission Spectroscopy (ETS) and found three resonances at the attachment energies of $0.11,2.8 \mathrm{eV}$, and $4.2 \mathrm{eV}$. Already qualitative MO arguments permitted their assignment as temporary electron attachment to the $\pi_{1}^{*}$ (inphase combination $\pi_{\mathrm{C}=\mathrm{C}}^{*}$ and the out-of-plane $\pi_{\mathrm{C} \equiv \mathrm{N}}^{*}$ ), $\pi_{2}^{*}$ (essentially the in-plane $\pi_{\mathrm{C} \equiv \mathrm{N}}^{*}$ ), and $\pi_{3}^{*}$ (out-of-phase combination $\pi_{\mathrm{C}=\mathrm{C}}^{*}$ and the out-of-plane $\pi_{\mathrm{C} \equiv \mathrm{N}}^{*}$ ). We observe these three resonances as enhancements of vibrational excitation cross sections shown in Fig. 1. The diagrams of self-consistent field (SCF) virtual orbitals shown in Fig. 2 confirm the assignment.

A very broad enhancement peaking around $6.3 \mathrm{eV}$ in the cross section for exciting the $\mathrm{C}-\mathrm{H}$ stretch vibration is similar to that observed already in the pioneering work on ethene ${ }^{16}$ and later in all compounds containing the $\mathrm{C}-\mathrm{H}$ bond, for example, in hydrocarbons, ${ }^{17}$ in particular cyclopropane, ${ }^{18}$ and in tetrahydrofurane. ${ }^{5}$

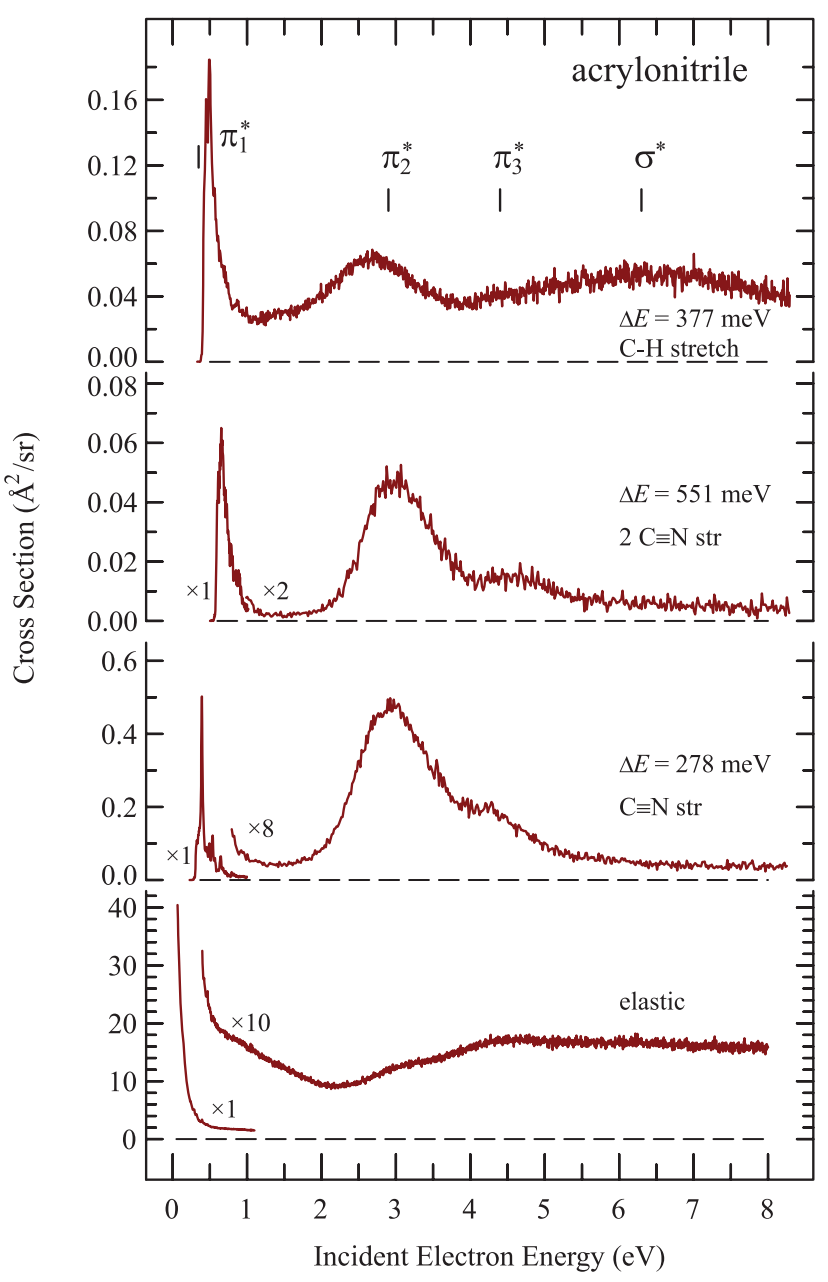

FIG. 1. Elastic and VE cross sections of acrylonitrile, recorded as a function of the incident electron energy, at a scattering angle of $135^{\circ}$.

It is assigned to broad overlapping $\sigma^{*}$ shape resonances. Burrow et al. observed a resonance at $5.2 \mathrm{eV}$ in ETS, which is narrower and probably has an origin in a core excited resonance. ${ }^{15}$

Figure 1 shows also the elastic cross section. It rises sharply at very low energies as expected for a molecule with a

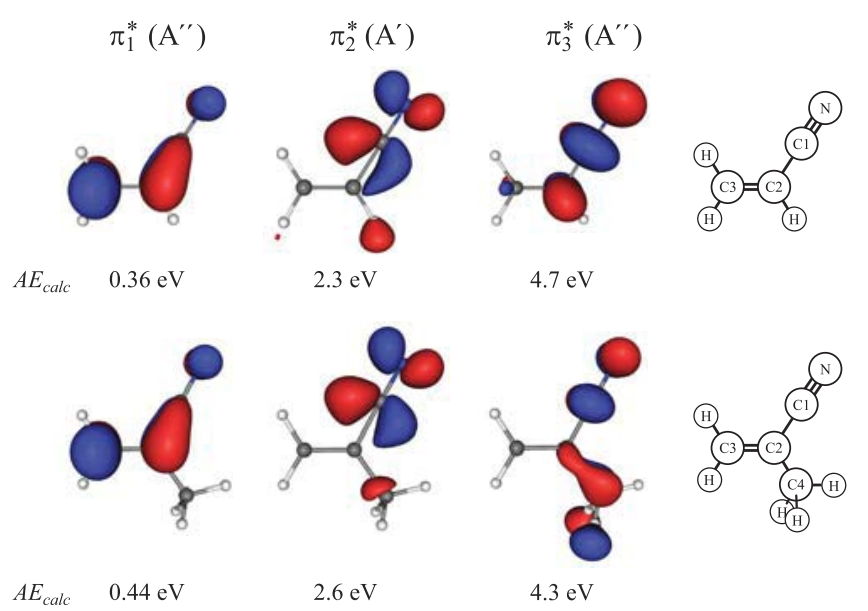

FIG. 2. Selected SCF 6-31G* orbitals of acrylonitrile (top) and methacrylonitrile (bottom), and the vertical attachment energies $V A E_{c a l c}$ estimated by the scaled Koopmans' theorem. ${ }^{11}$ Structures and numbering of the carbon atoms are given on the right. Drawn with the program Molekel. ${ }^{12}$ 


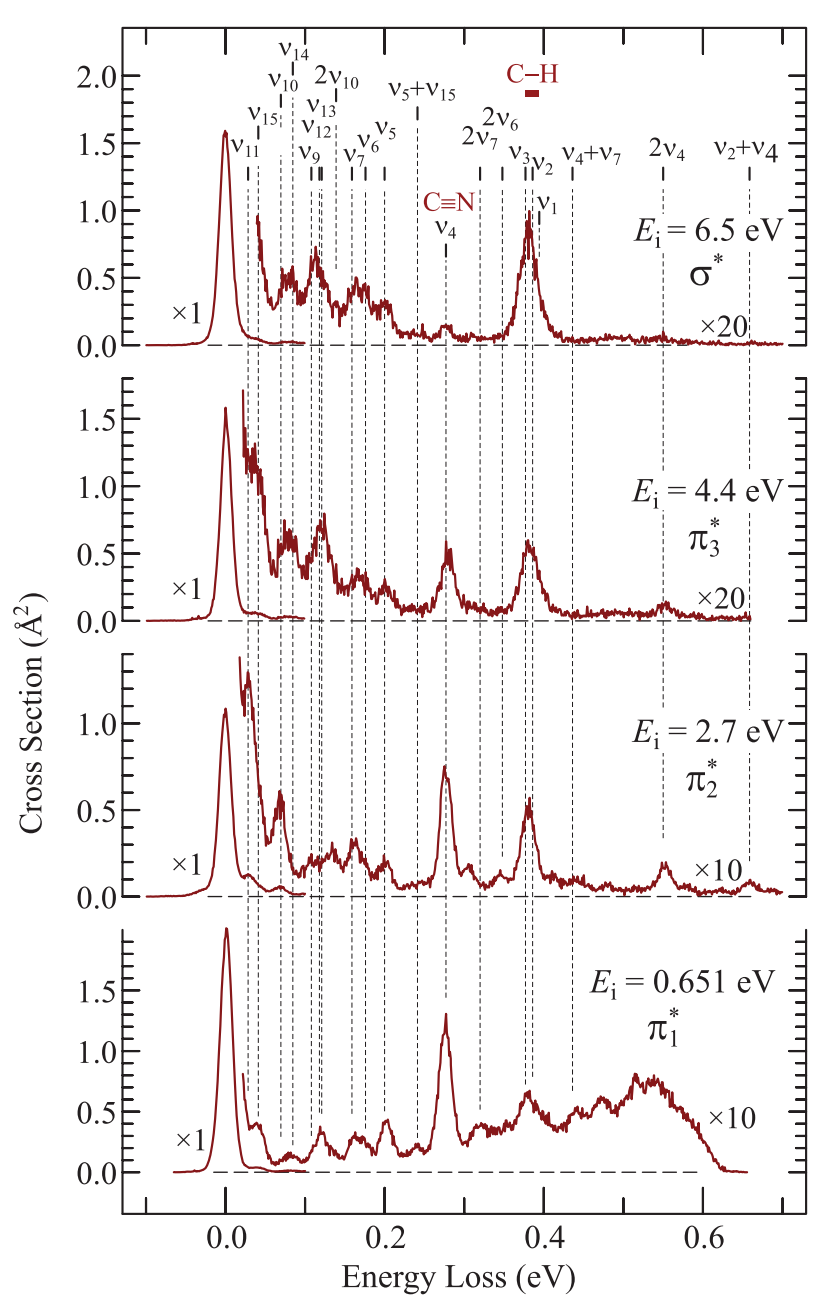

FIG. 3. Electron energy-loss spectra of acrylonitrile recorded with the constant incident energies indicated.

large dipole moment $\left(3.86 \mathrm{D}^{19}\right)$. The 2.8 and $4.4 \mathrm{eV}$ resonances cause shallow humps in the elastic cross section.

The selectivity with respect to which vibrational modes are excited by the four resonances is illustrated in Fig. 3.
Vibrational frequencies are listed in Table I. C-H stretch excitations $v_{2}$ and $v_{3}$ dominate the spectrum with $E_{i}=6.5 \mathrm{eV}$ and are caused by the very broad $\sigma^{*}$ shape resonances, antibonding with respect to the $\mathrm{C}-\mathrm{H}$ bonds. The non-totally symmetrical $\mathrm{CH}_{2}$ stretch vibration $v_{1}$ is not prominent, as expected. $\mathrm{C} \equiv \mathrm{N}$ stretch excitation $v_{4}$ is prominent in the spectra with incident energies corresponding to all three $\pi^{*}$ resonances, in line with the expectation based on the antibonding character of the $\pi^{*}$ orbitals with respect to the $\mathrm{C} \equiv \mathrm{N}$ bond illustrated in Fig. 2. The $\mathrm{C}=\mathrm{C}$ stretch vibration $v_{4}$ is excited at all four incident energies, somewhat surprisingly in view of the fact that the $\pi_{2}^{*}$ resonance does not nominally extend over the $\pi_{\mathrm{C}=\mathrm{C}}^{*}$ orbital. The in-plane rocking motion of the $\mathrm{C}-\mathrm{H}$ hydrogen $v_{7}$ is visible at the energies of the $\pi_{1}^{*}$ and $\pi_{2}^{*}$ resonances, surprisingly because it is not linked to antibonding properties of the $\pi^{*}$ orbitals. $v_{12}$ and $v_{13}$ (whose frequencies are too close to each other to be resolved) are visible at 0.651 and $4.4 \mathrm{eV}$, but not at $2.7 \mathrm{eV}$. Computer animation of the modes reveals that these two modes involve pyramidalization motions on the $\mathrm{C} 2$ and C3 carbon atoms (see Fig. 2 for numbering of atoms), and are, as already indicated in our earlier paper, ${ }^{1}$ driven by the fact that the two $\pi^{*}$ states of the anion are "on the verge" of becoming pyramidal, as indicated by the dramatic lowering of the two vibrational frequencies in the anion (Table I). In this respect, it is understandable that they are less prominent in the $E_{i}=2.7 \mathrm{eV}$ spectrum, because the $\pi_{2}^{*}$ orbital does not extend over the $\mathrm{C}=\mathrm{C}$ bond. Pronounced propensity is found for the excitation of the $\mathrm{C}=\mathrm{C}$ torsion vibration $v_{14}$, presumably driven by vibronic coupling of the $\pi_{\mathrm{C}=\mathrm{C}}^{*}$ and $\sigma^{*}$ orbitals, and therefore understandably missing at $2.7 \mathrm{eV}$, again because the $\pi_{2}^{*}$ orbital does not extend over the $\mathrm{C}=\mathrm{C}$ bond. In contrast, the excitation of the in-plane $\mathrm{C}=\mathrm{C}-\mathrm{C}$ bend vibration $v_{10}$ is prominent at $2.7 \mathrm{eV}$ because temporary occupation of the in-plane $\pi_{\mathrm{C} \equiv \mathrm{N}}^{*}$ orbital is likely to change the $\mathrm{C}=\mathrm{C}-\mathrm{C}$ angle. Similar argument may be forwarded for the excitation of the in-plane $\mathrm{C}-\mathrm{C} \equiv \mathrm{N}$ bend vibration $v_{11}$, prominent at $2.7 \mathrm{eV}$. In contrast, the excitation of the out-of-plane $\mathrm{C}-\mathrm{C} \equiv \mathrm{N}$ bend vibration $v_{15}$ by temporary occupation of the in-plane $\pi_{2}^{*}$ orbital is not

TABLE I. Observed ${ }^{13}$ and calculated ${ }^{1}$ (scaled by a factor of 0.97 ) vibrational frequencies of acrylonitrile and its anion (in $\mathrm{meV}$ ).

\begin{tabular}{|c|c|c|c|c|c|}
\hline \multirow[b]{2}{*}{ No. } & \multirow[b]{2}{*}{ Sym. } & \multirow[b]{2}{*}{ Type } & \multicolumn{2}{|c|}{ Neutral } & \multirow{2}{*}{$\begin{array}{c}\text { Anion } \\
\text { Calculated }\end{array}$} \\
\hline & & & Observed & Calculated & \\
\hline$v_{1}$ & $A^{\prime}$ & $(\mathrm{C}-\mathrm{H})$ asym. str of $\mathrm{CH}_{2}$ & 387 & 392 & 387 \\
\hline$v_{2}$ & & $(\mathrm{C}-\mathrm{H})$ str of $\mathrm{C}-\mathrm{H}$ & 382 & 378 & 377 \\
\hline$v_{3}$ & & $(\mathrm{C}-\mathrm{H})$ sym. str of $\mathrm{CH}_{2}$ & 377 & 382 & 376 \\
\hline$v_{4}$ & & $\mathrm{C} \equiv \mathrm{N}$ str & 278 & 280 & 257 \\
\hline$v_{5}$ & & $\mathrm{C}=\mathrm{C}$ str & 200 & 201 & 179 \\
\hline$v_{6}$ & & $\mathrm{CH}_{2}$ scissoring & 176 & 174 & 168 \\
\hline$v_{7}$ & & $\mathrm{C}-\mathrm{H}$ rock & 159 & 159 & 146 \\
\hline$v_{8}$ & & $\mathrm{CH}_{2}$ rock & 136 & 134 & 131 \\
\hline$v_{9}$ & & $\mathrm{C}-\mathrm{C}$ str & 108 & 106 & 107 \\
\hline$v_{10}$ & & $\mathrm{C}=\mathrm{C}-\mathrm{C}$ bend & n.o. & 70.2 & 72.0 \\
\hline$v_{11}$ & & $\mathrm{C}-\mathrm{C} \equiv \mathrm{N}$ bend & 28.4 & 29.2 & 28.9 \\
\hline$v_{12}$ & $A^{\prime \prime}$ & $\mathrm{C}-\mathrm{H}$ wag (C2 pyram) & 121 & 121 & 69.5 \\
\hline$v_{13}$ & & $\mathrm{CH}_{2}$ wag (C3 pyram) & 118 & 120 & 24.7 \\
\hline$v_{14}$ & & $\mathrm{C}=\mathrm{C}$ torsion $(\mathrm{C} 2$ pyram $)$ & 84.7 & 85.7 & 59.6 \\
\hline$v_{15}$ & & oop $\mathrm{C}-\mathrm{C} \equiv \mathrm{N}$ bend & 41.3 & 42.0 & 39.3 \\
\hline
\end{tabular}


observed, but it is clearly promoted by temporary occupation of the out-of-plane $\pi_{1}^{*}$ and $\pi_{3}^{*}$ orbitals.

\section{B. Methacrylonitrile}

An overview of the resonances revealed by the VE cross sections is shown in Fig. 4. Qualitatively, one would expect the $\pi_{3}^{*}$ orbital to be an out-of-phase combination of the $\pi_{\mathrm{C}=\mathrm{C}}^{*}$ and the $\pi_{\mathrm{C} \equiv \mathrm{N}}^{*}$, but the SCF virtual molecular orbitals in Fig. 2 make the interesting prediction that $\pi_{3}^{*}$ is dominated by hyperconjugation with the pseudo- $\pi$ orbital of the $\mathrm{C}-\mathrm{CH}_{3}$ moiety and does not have any coefficient on the $\mathrm{CH}_{2}$ carbon.

Table II lists the frequencies and the types of the individual modes and the selectivity of vibrational excitation is illustrated in Fig. 5. Unlike Fig. 3, the spectra were recorded with constant residual energies, but this circumstance is not significant; the incident energies were in energy ranges within the $\pi_{1}^{*}, \pi_{2}^{*}$, and $\pi_{3}^{*}$ resonances. As in the case of acrylonitrile, the $\mathrm{C} \equiv \mathrm{N}$ stretch vibration $v_{5}$ is more prominent in the lower three spectra, in line with the assignment of the resonances as $\pi_{\mathrm{C} \equiv \mathrm{N}}^{*}$.

The $\mathrm{C}=\mathrm{C}$ stretch vibration $v_{6}$ is prominent in $\pi_{1}^{*}$ but weak in $\pi_{2}^{*}$ and $\pi_{3}^{*}$, in line with the facts that $\pi_{2}^{*}$ is primarily the in-plane $\pi_{\mathrm{C} \equiv \mathrm{N}}^{*}$ and $\pi_{3}^{*}$ somewhat surprisingly does not have any $\pi_{\mathrm{C}=\mathrm{C}}^{*}$ contribution (Fig. 2). The excitation of $v_{11}\left(\mathrm{CH}_{3}\right.$ rock), not clearly distinguishable from $v_{19}\left(\mathrm{CH}_{3} \mathrm{wag}\right)$, is quite prominent in the $\pi_{1}^{*}$ resonance $\left(E_{i}=0.15\right.$ and $\left.0.35 \mathrm{eV}\right)$ and

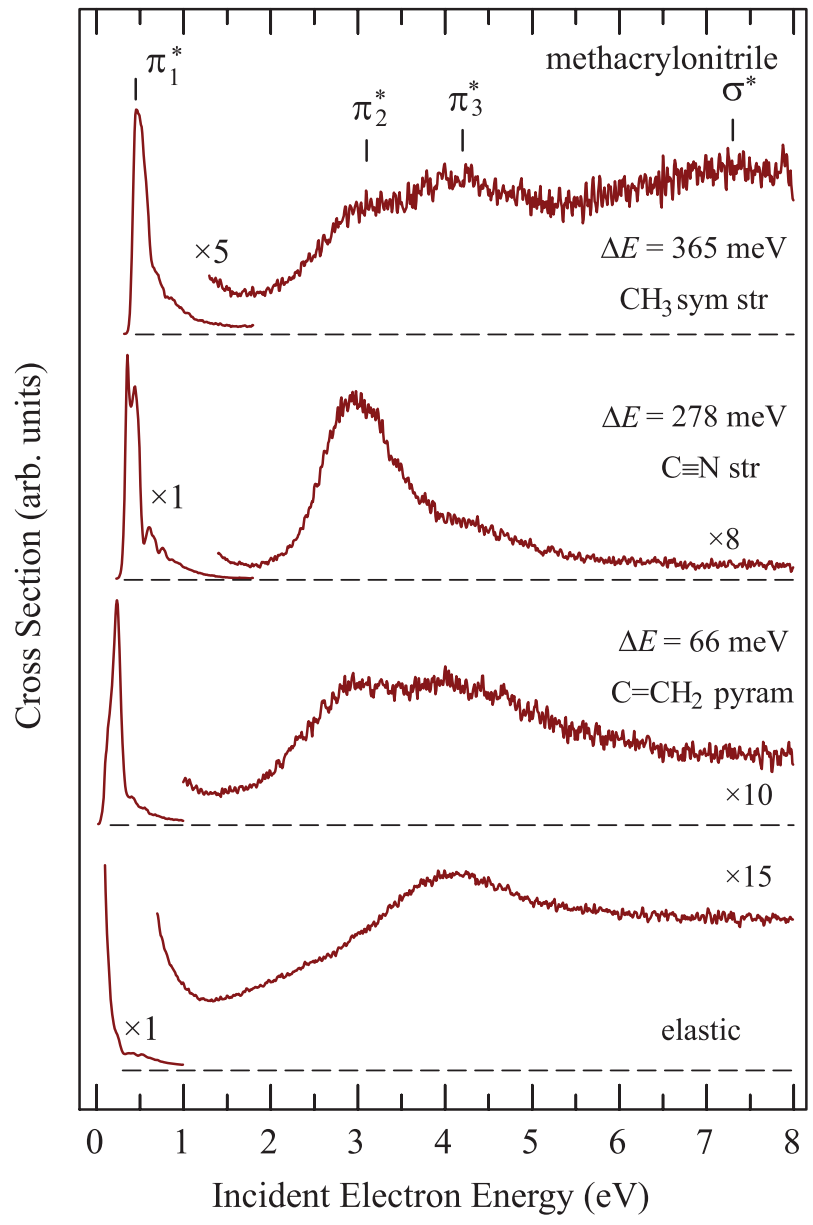

FIG. 4. Energy-dependence spectra of methacrylonitrile measured at a scattering angle of $135^{\circ}$. is similarly surprising as the excitation of $v_{7}(\mathrm{C}-\mathrm{H}$ rock $)$ in acrylonitrile mentioned above. Finally, the excitation of $v_{22}$ and $v_{23}$ (both out-of-plane $\mathrm{C}-\mathrm{C} \equiv \mathrm{N}$ bends) is quite prominent, not surprisingly because both frequencies are lowered in the anion (Table II).

The $\pi_{3}^{*}$ resonance is nearly absent in the $\mathrm{C}-\mathrm{H}$ stretch excitation function of acrylonitrile in Fig. 1 but clearly visible in $\mathrm{C}-\mathrm{H}$ stretch excitation function of methacrylonitrile in Fig. 4, presumably a consequence of the hyperconjugation of the $\pi_{3}^{*}$ orbital with the $\mathrm{CH}_{3}$ group, visible in Fig. 2.

\section{DETAILS OF THE LOWEST RESONANCE}

\section{A. 2D spectrum of acrylonitrile}

Fig. 6 shows the 2D spectrum of acrylonitrile. It is identical to that given in the earlier paper, ${ }^{1}$ except that the elastic signal, as a function of energy, is shown normalized to the experimental elastic value, which decreases with energy as shown in the bottom trace of Fig. 1, and not to a constant as in the earlier paper. This has the advantage of more realistic signal intensities, albeit at the price of reduced visibility of weaker signals at higher incident energies.

The 2D EEL spectrum plots the differential cross section (DCS), represented by color, as a function of the electron energy loss $\Delta E$ and of the incident electron energy $E_{i}$. The elastic peak has $\Delta E=0$ and is represented by the vertical "elastic ridge" on the left. The vertical cut of the $2 \mathrm{D}$ spectrum along this "ridge" is the energy-dependence of the elastic cross section, shown (in absolute units) by the lowest curve in Fig. 7. The ordinate of the 2D spectrum defines the vibrational states of the temporary negative ion, that is, the resonances. The abscissa defines the final states of the neutral molecule. The assignments of specific final vibrational states and resonances given in Fig. 6 are taken from our earlier paper. ${ }^{1}$

The largest possible $\Delta E$ is equal to $E_{i}$ for each incident energy and corresponds to the situation where all the energy of the incident electron is used for internal excitation of the acrylonitrile target and the electron leaves with nearly zero kinetic energy. At this point, the excitation of the final state is at its threshold and this situation is encountered along the diagonal labeled "threshold line" in Fig. 6.

Some of the prominent excitations, in particular, those of $v_{5}$ and $v_{6}$ can be easily understood in terms of the antibonding properties of the temporarily occupied $\pi^{*}$ orbital, but others cannot, particularly $v_{7}\left(\mathrm{C}-\mathrm{H}\right.$ rock), $v_{10}$ (in-plane $\mathrm{C}=\mathrm{C}-\mathrm{C}$ bend), and $v_{6}\left(\mathrm{CH}_{2}\right.$ scissoring), although they are among the strongest inelastic features in the $2 \mathrm{D}$ spectrum.

A vertical cut of the $2 \mathrm{D}$ spectrum at a given energy loss corresponding to a given final state is the energy-dependence of VE cross section, with examples shown in Figs. 7 and 8. A horizontal cut through the $2 \mathrm{D}$ spectrum is an energyloss spectrum recorded at a given constant incident energy $E_{i}$, corresponding to a given resonance. An example is the lowest curve in Fig. 3.

As detailed in our earlier paper, an expectation for the 2D spectrum would be vertical patterns of dots, corresponding to various resonances exciting a given final state, and horizontal patterns of dots, corresponding to a given resonance decaying 
TABLE II. Observed ${ }^{14}$ and calculated (B3LYP/6-311++ G(2df,2p), scaled by a factor of 0.97) vibrational frequencies of methacrylonitrile and its anion (in $\mathrm{meV}$ ).

\begin{tabular}{|c|c|c|c|c|c|}
\hline \multirow[b]{2}{*}{ No. } & \multirow[b]{2}{*}{ Sym. } & \multirow[b]{2}{*}{ Type } & \multicolumn{2}{|c|}{ Neutral } & \multirow{2}{*}{$\begin{array}{c}\text { Anion } \\
\text { Calculated }\end{array}$} \\
\hline & & & Observed & Calculated & \\
\hline$v_{1}$ & \multirow[t]{16}{*}{$A^{\prime}$} & $\mathrm{CH}_{2}$ asym str & 386 & 391 & 387 \\
\hline$v_{2}$ & & $\mathrm{CH}_{2}$ sym str & 377 & 380 & 375 \\
\hline$v_{3}$ & & $\mathrm{CH}_{3}$ asym str & 372 & 375 & 368 \\
\hline$v_{4}$ & & $\mathrm{CH}_{3}$ sym str & 365 & 366 & 349 \\
\hline$v_{5}$ & & $\mathrm{C} \equiv \mathrm{N}$ str & 276 & 279 & 256 \\
\hline$v_{6}$ & & $\mathrm{C}=\mathrm{C}$ str & 202 & 202 & 181 \\
\hline$v_{7}$ & & $\mathrm{CH}_{3}$ asym def & 182 & 179 & 178 \\
\hline$v_{8}$ & & $\mathrm{CH}_{2}$ scissoring & 174 & 174 & 164 \\
\hline$v_{9}$ & & $\mathrm{CH}_{3}$ umbrella & 171 & 170 & 167 \\
\hline$v_{10}$ & & $\mathrm{C}-\mathrm{CH}_{3}$ str & 157 & 155 & 154 \\
\hline$v_{11}$ & & $\mathrm{CH}_{3}$ rock & 126 & 125 & 122 \\
\hline$v_{12}$ & & $\mathrm{CH}_{2}$ rock & 118 & 116 & 115 \\
\hline$v_{13}$ & & $\mathrm{C}-\mathrm{CN}$ and $\mathrm{C}-\mathrm{CH}_{3}$ str & 93.0 & 90.9 & 91.3 \\
\hline$v_{14}$ & & in plane $\mathrm{C}-\mathrm{C} \equiv \mathrm{N}$ bend & 72.2 & 73.6 & 76.2 \\
\hline$v_{15}$ & & $\mathrm{CH}_{3}-\mathrm{C}=\mathrm{C}_{2}$ bend & 46.9 & 46.1 & 43.5 \\
\hline$v_{16}$ & & $\mathrm{C}-\mathrm{C} \equiv \mathrm{N}$ bend & 25.0 & 24.5 & 24.8 \\
\hline$v_{17}$ & \multirow[t]{8}{*}{$A^{\prime \prime}$} & $\mathrm{CH}_{3}$ asym str & 369 & 373 & 339 \\
\hline$v_{18}$ & & $\mathrm{CH}_{3}$ asym def & 180 & 180 & 176 \\
\hline$v_{19}$ & & $\mathrm{CH}_{3}$ wag & 130 & 127 & 124 \\
\hline$v_{20}$ & & $\mathrm{CH}_{2}$ wag (pyram on $\mathrm{C} 3$ ) & 115 & 116 & 33.6 \\
\hline$v_{21}$ & & $\mathrm{C}=\mathrm{C}$ torsion & 91.4 & 90.8 & 51.1 \\
\hline$v_{22}$ & & oop $\mathrm{C}-\mathrm{C} \equiv \mathrm{N}$ bend & 66.0 & 67.9 & 61.2 \\
\hline$v_{23}$ & & oop $\mathrm{C}-\mathrm{C} \equiv \mathrm{N}$ bend & 33.8 & 34.9 & 28.9 \\
\hline$v_{24}$ & & $\mathrm{CH}_{3}$ torsion & 30.6 & $\ldots$ & 12.3 \\
\hline
\end{tabular}

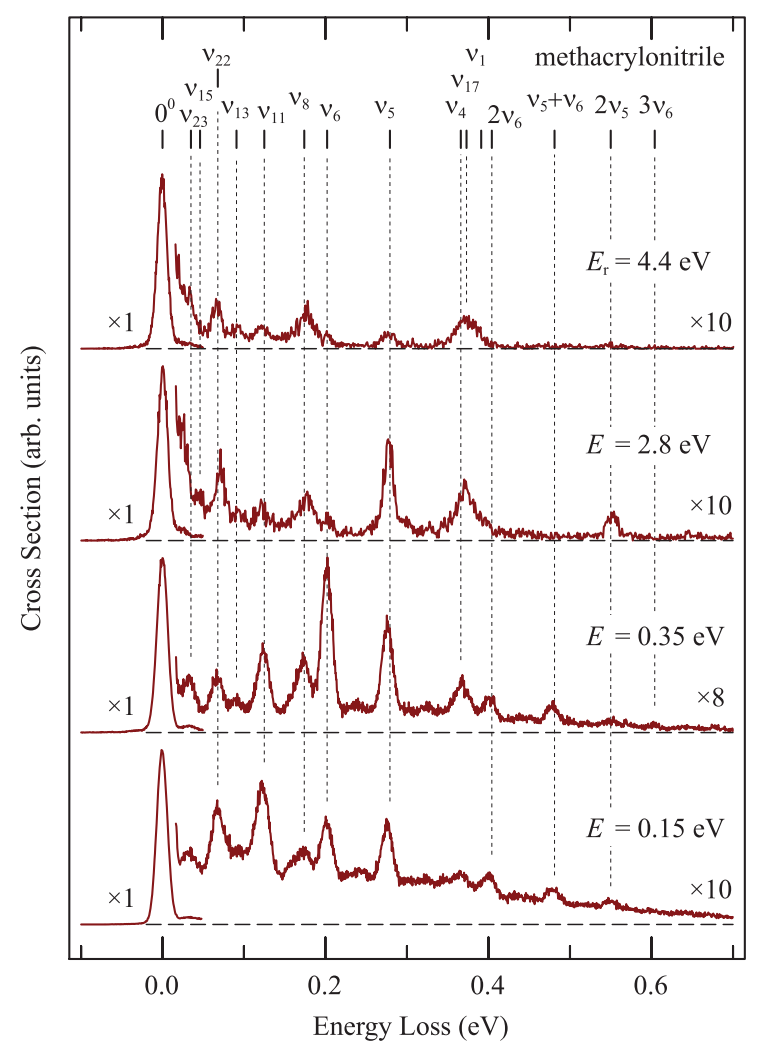

FIG. 5. Energy-loss spectra recorded with constant residual energies chosen such that the incident energies are within the $\pi_{1}^{*}\left(E_{r}=0.15\right.$ and $\left.0.35 \mathrm{eV}\right), \pi_{2}^{*}$ $\left(E_{r}=2.8 \mathrm{eV}\right)$, and $\pi_{3}^{*}\left(E_{r}=4.4 \mathrm{eV}\right)$ resonances in methacrylonitrile. to various final states. The surprising result reported in the earlier paper was the diagonal patterns, labeled $A, B$, and $C$ in Fig. 6. The diagonal patterns explain certain striking observations in the energy loss spectra. Thus, the high intensity of the $\mathrm{C} \equiv \mathrm{N}$ stretch peak in the bottom trace in Fig. 3 is seen to be a manifestation of an enhanced excitation along the diagonal $C$ in Fig. 6 and appears as a blue spot at the coordinates $\Delta E$ $=278 \mathrm{meV}$ and $E_{i}=651 \mathrm{meV}$ there. This spot appears also as the peak labeled $4^{2}$ at $E_{i}=651 \mathrm{meV}$ in the top curve of Fig. 8.

The quantity common to all spots lying on one of the diagonals $A, B$, and $C$ in Fig. 6 is the residual energy $E_{r}$. An insight about the nature of the excitations on a given diagonal is thus revealed by recording a cut on that diagonal, which is an energy-loss spectrum recorded with a given $E_{r}$, such as given in Fig. 9.

The schematic potential curves in Figure 10, where the abscissa corresponds qualitatively to the $v_{7}$ mode, show that a given residual energy is associated with a given change of vibrational quantum $\Delta n_{j}=n_{j}-n_{j}^{\prime}$, the lowest $E_{r}$ being associated with $\Delta n_{j}=0$ for all $n_{j}$ and even more importantly, for all modes $j$. The residual energy associated with $\Delta n_{j}=0$ thus depends only on $A E_{a}$ and not on which vibrational mode is concerned. These transitions are arranged along the diagonal $A$ in Figure 6. This explains why there are so many dots on the diagonal $A-$ all modes $j$ have their $\Delta n_{j}=0$ peaks on this diagonal. When the detachment is regarded as a half-collision, then the detachment could be called "vibrationally elastic" along the diagonal $A$, since $n_{j}$ and $n_{j}^{\prime}$ are equal. 


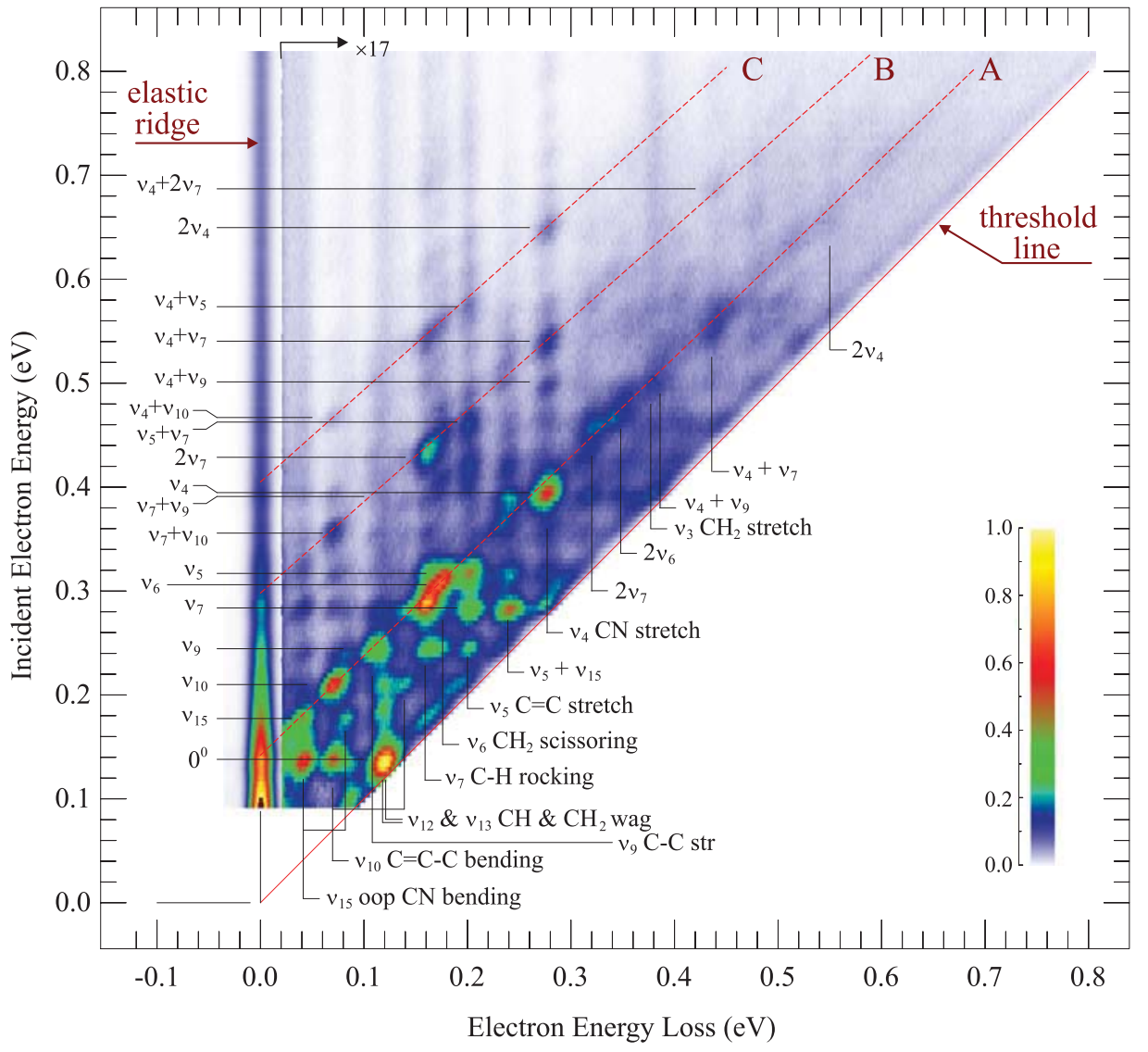

FIG. 6. Two-dimensional electron energy loss spectrum of acrylonitrile. The inelastic signal at $\Delta E>0.02 \mathrm{eV}$ is shown multiplied $17 \times$.

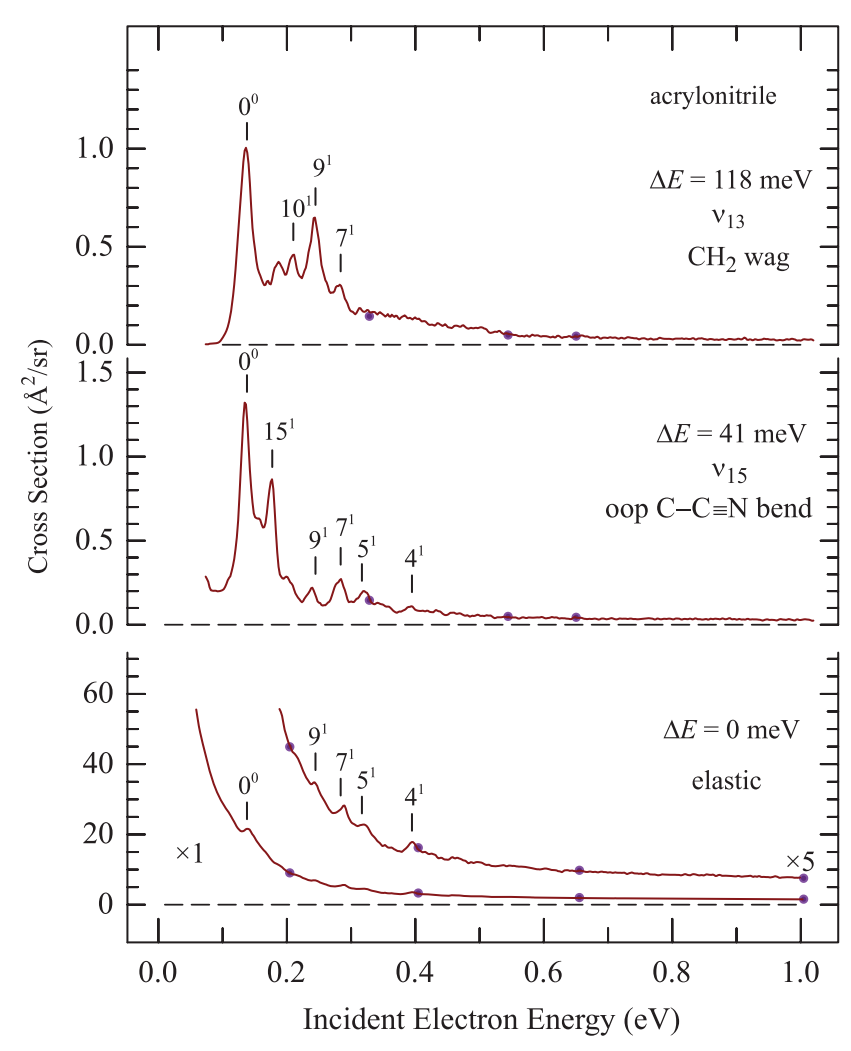

FIG. 7. Low energy detail of the elastic and VE cross sections of acrylonitrile, recorded as a function of the incident electron energy. The circles indicate separately measured absolute cross section values to which the red curves were normalized.
Figure 10 shows that this diagonal, extrapolated to $\Delta E$ $=0$, crosses the "elastic ridge" at $E_{i}=A E_{a}$ and this expectation is confirmed by the experiment in Figure 6. This extrapolation represents a way of determining $A E_{a}$, found to be $A E_{a}=138 \mathrm{meV}$ for acrylonitrile. Figure 10 further shows that $E_{r}$ should decrease slowly with increasing quanta, the rate of decrease being proportional to the difference of the neutral and anion vibrational energies $\Delta v=v-v^{\prime}$. The diagonal $A$ should thus slowly approach the threshold line - an expectation confirmed by the experiment.

The diagonal $B$ is specific for one normal mode, presumably $v_{7}$. It is associated with $\Delta v_{7}=-1$ and the residual energy is $E_{r}=A E_{a}+v_{7}$ for all features. Prominent peaks on this diagonal are $v_{7}+v_{10} \rightarrow v_{10}, 2 v_{7} \rightarrow v_{7}, v_{4}+v_{7} \rightarrow v_{4}$, and $v_{4}$ $+2 v_{7} \rightarrow v_{4}+v_{7}$. The diagonal $C$ is characterized by $\Delta v_{4}=-1$, with prominent peaks $v_{4}+v_{10} \rightarrow v_{10}, v_{4}+v_{7} \rightarrow v_{7}$, and $2 v_{4}$ $\rightarrow v_{4}$. More diagonals can be discerned, for example, with $\Delta v_{9}=-1$, but they are not labeled in Figure 6 .

\section{B. Excitation functions in acrylonitrile}

Figs. 7 and 8 show the more conventional form of cross section presentation, as excitation functions of the elastic peak and of selected vibrations. They are vertical cuts through the 2D spectrum of Fig. 6, recorded at the respective energy losses indicated in Figs. 7 and 8 and complement the information of the $2 \mathrm{D}$ spectrum by showing the absolute cross section values.

As already pointed out by Burrow et al., ${ }^{15}$ the resonant structures in acrylonitrile are narrow, around $17.5 \mathrm{meV}$ in our 


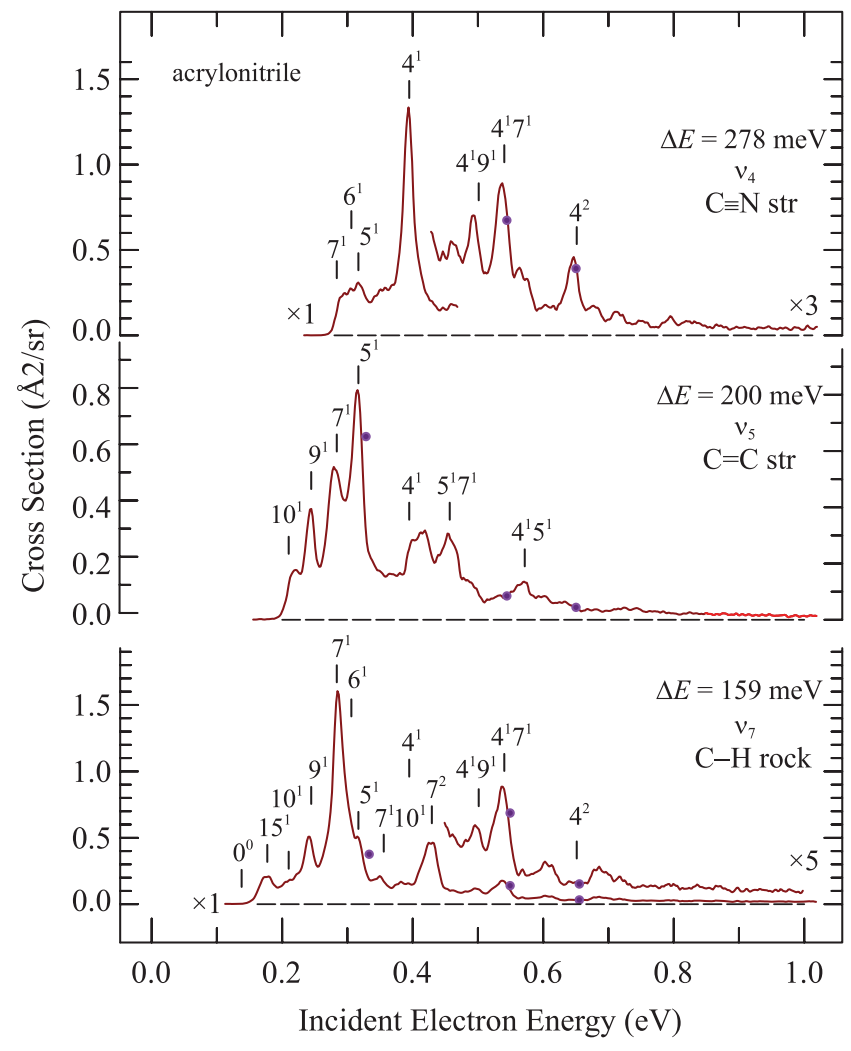

FIG. 8. VE cross sections of acrylonitrile, continued from Fig. 7.

spectra, only marginally wider than the instrumental resolution of $11 \mathrm{meV}$, implying a lifetime determined width of about $14 \mathrm{meV}$.

The initial expectation of the appearance of the excitation functions would be that they show progressions of peaks corresponding to vibrational levels of the resonance, and that one observes the same resonant peaks in various final levels. The observed cross sections in Figs. 7 and 8 do not correspond to these expectations - they show seemingly irregular peaks, at different positions in each final channel, without discernible progressions. In fact, the assignment of the resonant peaks was possible only with the help of the $2 \mathrm{D}$ spectrum. The 2D spectrum shows why this is the case - it is because the intensities of the peaks are not enhanced along progressions of particular modes but along the diagonals $A, B$, and $C$. Which resonant peak is intense in Figs. 7 and 8 is thus determined by whether they do or do not lie on one of the diagonals in Fig. 6. Examples are the $7^{1}$ resonant peak in the excitation function of the $v_{7}$ vibration (bottom curve in Fig. 8), the $5^{1}$ resonant peak in the excitation function of the $v_{5}$ vibration (center curve in Fig. 8), the $4^{1}$ resonant peak in the excitation function of the $v_{4}$ vibration (top curve in Fig. 8), and the $15^{1}$ resonant peak in the excitation function of the $v_{15}$ vibration (center curve in Fig. 7). The top curve in Fig. 7 appears to be an unexplained exception to this rule, the strongest peak (apart from the origin $0^{0}$ ) is the $9^{1}$ resonance in the excitation of the $v_{13}$ vibration. Sharp structures are observed in the elastic cross section in the bottom curve in Fig. 7, the most prominent being those due to the $0^{0}, 7^{1}$, and $4^{1}$ resonances.

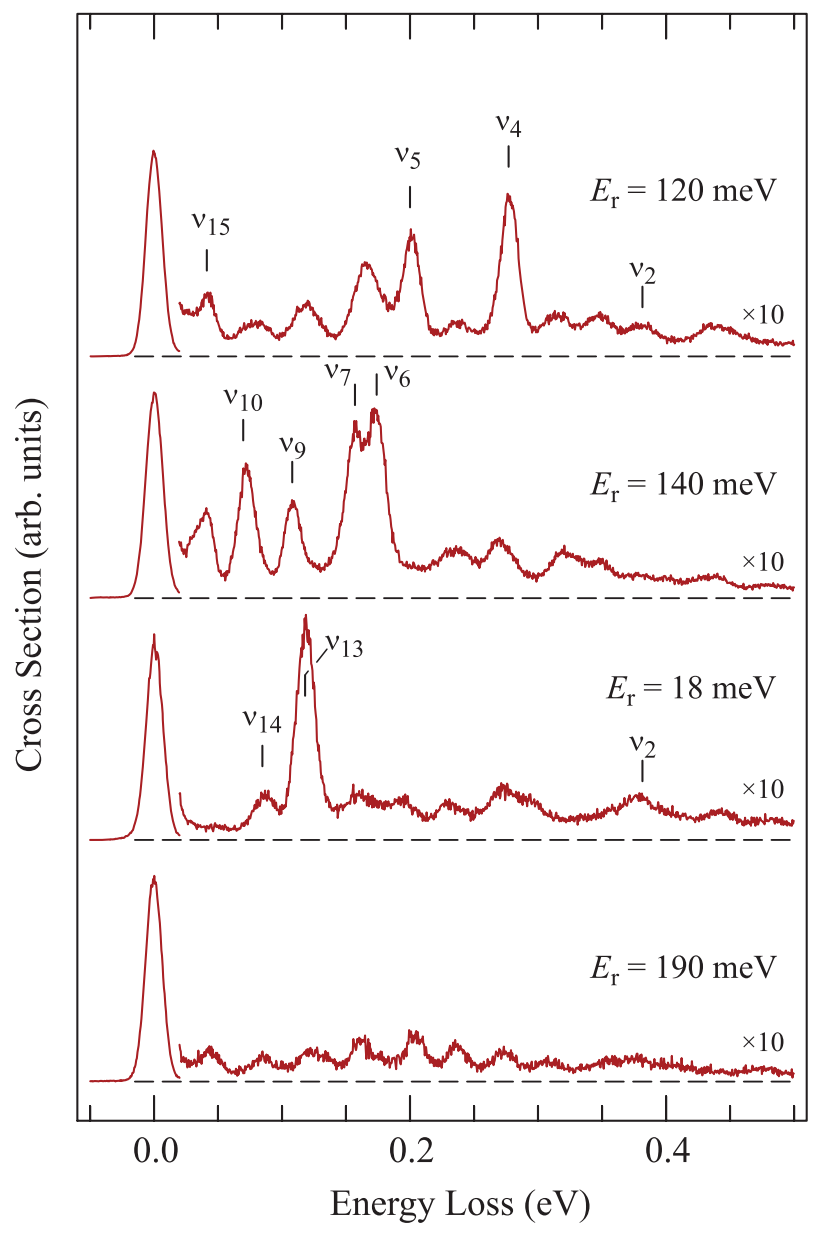

FIG. 9. Electron energy-loss spectra of acrylonitrile recorded with the constant residual energies indicated.

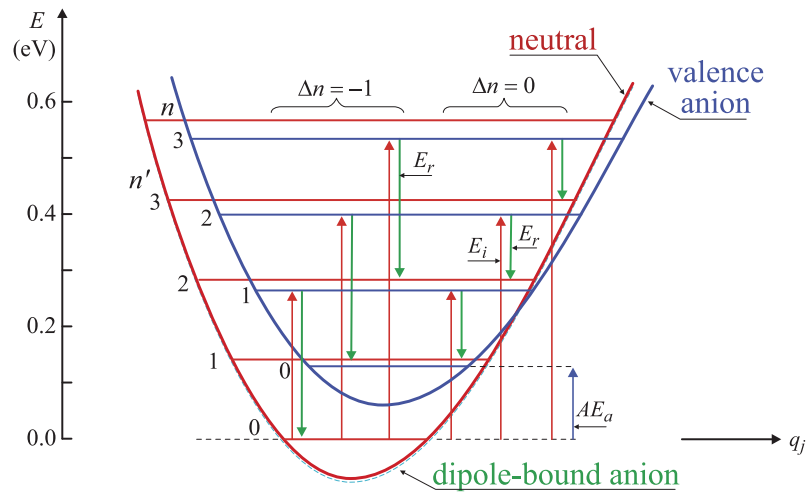

FIG. 10. Schematic potential curves of the neutral molecule (red), the valence anion (shape resonance, blue), and the dipole-bound anion (green dashed). ${ }^{1}$ The vibrational quantum is approximately $v_{7}$. The adiabatic electron attachment energy $A E_{a}$ is indicated at the bottom right. Electron attachment is shown by red upward arrows, electron detachment by green downpointing arrows. The scattering events (attachment-detachment) are grouped into those where the vibrational quantum number does not change during detachment (marked $\Delta n=0$ on the top) and those where the vibrational quantum number decreases by one during detachment (marked $\Delta n=-1$ on the top). The $\Delta n=0$ events lie on the diagonal $A$, the $\Delta n=-1$ on the diagonal $B$ in Figs. 6 and 11. All $\Delta n=0$ events yield nearly the same residual energy $E_{r}$ (given by $A E_{a}$ ) and are thus selectively visualized in a spectrum recorded with a constant $E_{r}=A E_{a}$ in Figs. 9 and 13 . 


\section{Energy-loss spectra recorded along the diagonals}

Insight into the diagonal processes may be gained from energy-loss spectra recorded at residual energies corresponding to the diagonal features - they are shown in Fig. 9. The main diagonal, $A$, corresponds to the spectrum with $E_{r}$ $=138 \mathrm{meV}$ (the spectrum was recorded at $E_{r}=140 \mathrm{meV}$, but the difference is well within the resolution and is not relevant). It has intense peaks corresponding to $v_{6}, v_{7}, v_{9}$, and $v_{10}-$ in all these cases, the detachment is "vibrationally elastic," the vibrational state of the resonance enhancing the excitation is the same as the final vibrational state of the neutral molecule - the vibrational quantum does not change during the detachment.

Certain peaks are slightly below the main diagonal, when the vibrational frequency in the anion is notably lower than that of the neutral - such as the $\mathrm{C}=\mathrm{C}$ and $\mathrm{C} \equiv \mathrm{N}$ stretch vibrations $v_{4}$ and $v_{5}$. These vibrations are prominent in the spectrum recorded slightly below the diagonal, with $E_{r}=120 \mathrm{meV}$. The spectrum recorded with $E_{r}=190 \mathrm{meV}$ is between the diagonals $A$ and $B$. The vibrational peaks are much weaker than in the "on diagonal" spectra, and many peaks have comparable intensities - there is less selectivity in the excitation process. The spectrum recorded very close to threshold, at $E_{r}$ $=18 \mathrm{meV}$, has, as is already visible in the $2 \mathrm{D}$ spectrum in Fig. 6, an intense $v_{13}$ (and/or the unresolved $v_{12}$ ) peak, where the $0^{0}$ ground vibrational level of the $\pi_{1}^{*}$ resonance decays quasi-resonantly into a vibrational level lying at only very slightly lower energy and a very slow electron. The reason for this propensity is not known. The remaining peaks in the threshold spectrum are quite weak, in contrast to methacrylonitrile as will be shown below.

\section{2D spectrum of methacrylonitrile}

An overview of the resonant features and decay channels is given by the 2D spectrum in Fig. 11. The inelastic peaks are about $2 \times$ weaker relative to the elastic peak than in acrylonitrile. The lower intensity of vibrational excitation and the fact that the resonances are much broader indicate that the methyl group substantially shortens the resonance lifetime, making the features of the 2D spectrum vertically elongated. The lifetime-related width also prevents detailed assignment of the resonances to individual vibrational modes. The similarity of certain features with the 2D spectrum of acrylonitrile permits assignment in some cases, in particular $v_{6}, v_{5}$, and $2 v_{5}$, indicated on the left in Fig. 11.

The feature interesting in acrylonitrile - the diagonal patterns - can be discerned also in methacrylonitrile. In particular, the intense peaks in the excitations of $v_{23}$ (out-of-plane $\mathrm{C}-\mathrm{C} \equiv \mathrm{N}$ bending $), v_{6}(\mathrm{C}=\mathrm{C}$ stretch $)$, and $v_{5}(\mathrm{C} \equiv \mathrm{N}$ stretch $)$ lie on the diagonal $A$. There are more exceptions, however, in particular the peak at about $0.26 \mathrm{eV}$ in the excitation of $v_{11}$ and also peaks at the excitations of $2 v_{6}, v_{5}+v_{6}$, and $2 v_{5}$ are clearly below the diagonal $A$ (in fact. they lie on a diagonal of their own) and we cannot explain the reason for this. As explained above in connection with the $2 \mathrm{D}$ spectrum of acrylonitrile, a peak can lie below the diagonal if the frequency of a given mode is lower in the anion than in the neutral molecule. Table II indicates that this is dramatically the case for $v_{20}$ and to a

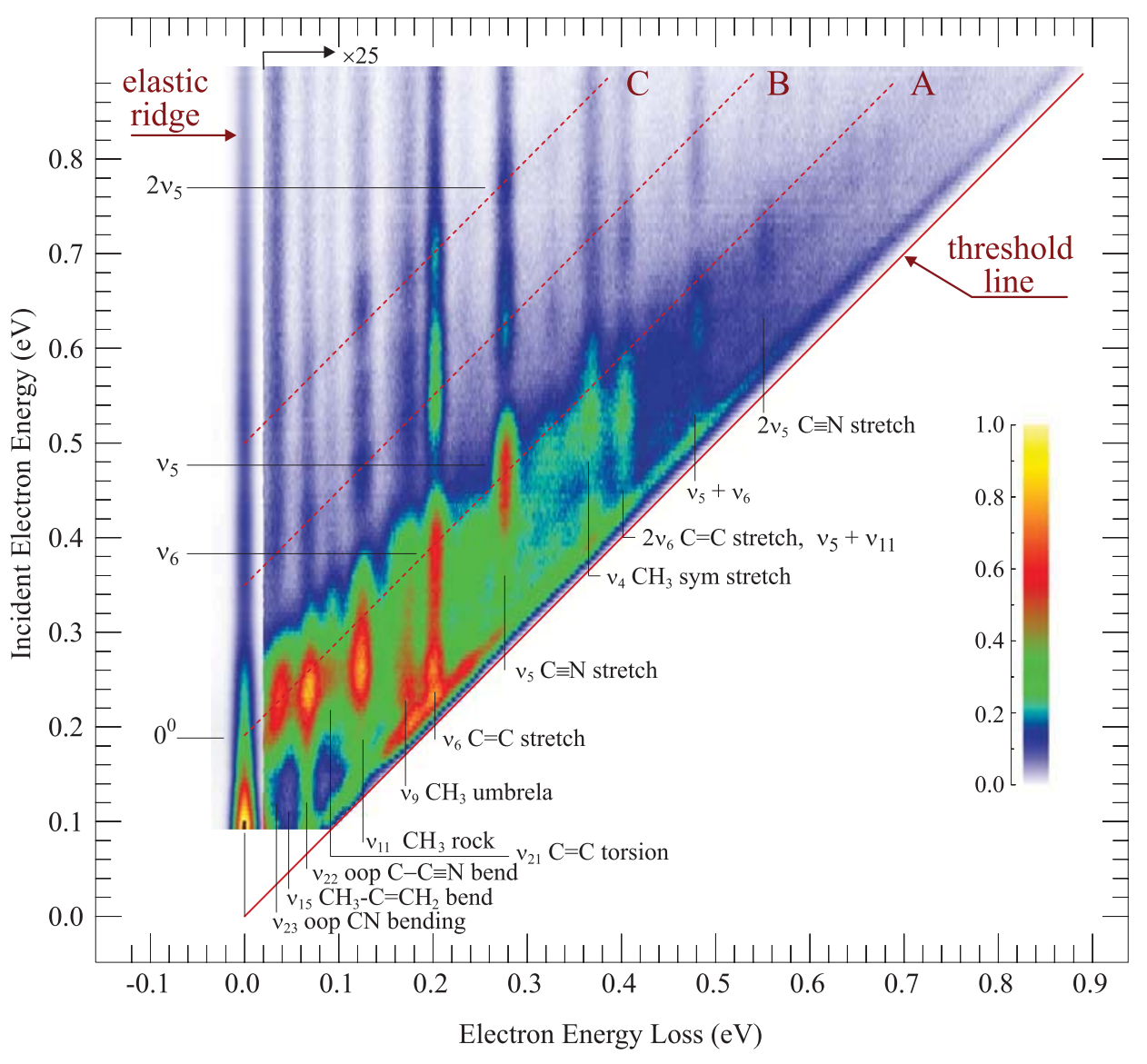

FIG. 11. 2D spectrum of methacrylonitrile. The inelastic signal at $\Delta E$ $>0.02 \mathrm{eV}$ is shown multiplied $25 \times$. 
lesser degree for $v_{21}$, but the frequencies are such that they cannot explain the shifts of the peaks away from the diagonal. It is not clear why there are more below-diagonal peaks in methacrylonitrile than in acrylonitrile.

Yet another observation is that the threshold signal, just above the "threshold line," is stronger in Fig. 11 than in Fig. 6. (The limited capability of the instrument to collect very slow electrons prevented measurement exactly on the threshold line.) This is a manifestation of the "unspecific" vibrational excitation at threshold and will be discussed in more detail below.

One feature seems present in both compounds - it is the propensity for exciting vibrations near threshold when a resonance can decay "quasi-resonantly," i.e., when there is a final state which has nearly the same energy as a given resonance, leading to a peak at the threshold line. In acrylonitrile, this propensity strongly enhanced the excitation of the (unresolved) $v_{13}$ and $v_{12}$ pair of peaks (Fig. 6). Similarly, in Fig. 11 the excitation of $v_{6}, v_{8}$, and also $v_{17}$ is exceptionally strong near threshold.

The diagonal $A$ intersects the "elastic ridge" at $190 \mathrm{meV}$, and this number represents an estimate of the adiabatic attachment energy - although the number is less well defined than in acrylonitrile because of the larger lifetime-given width. This number is, albeit only slightly, larger than the $138 \mathrm{meV}$ found for acrylonitrile, as expected - a destabilization of both $\pi$ and $\pi^{*}$ orbitals by alkyl substituents is a general phenomenon encountered in UV photoelectron and electron transmission spectroscopies.

\section{E. Excitation functions in methacrylonitrile}

Three examples of the excitation functions are shown in Fig. 12. The elastic cross section rises steeply at low energies because of the large dipole moment $\left(3.69 \mathrm{D}^{19}\right)$ of methacry-

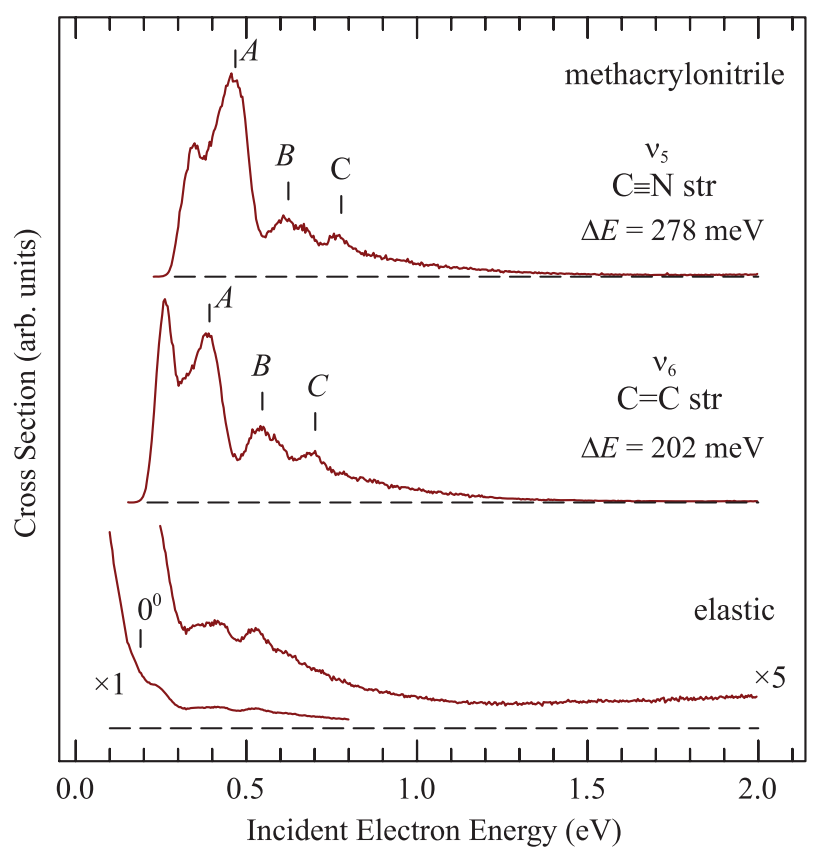

FIG. 12. Energy-dependence spectra of methacrylonitrile. The origin $0^{0}$ of the $\pi^{*}$ resonance and the positions of the $A, B$, and $C$ diagonals are indicated. lonitrile. The width of the resonant peaks indicates a lifetime determined width of the resonance of about $100 \mathrm{meV}$, about $7 \times$ wider than in acrylonitrile. It is natural for resonances to become wider when they are at higher energies because the relative height of the centrifugal barrier through which the electron tunnels out during the detachment is lower. It is also natural for resonances to become wider when the molecule is larger because it often means less symmetry and larger contribution of low partial waves (in particular s-wave) in the resonance through which the electron can "leak out." But the methacrylonitrile resonance energy, $190 \mathrm{meV}$, is only very slightly higher than that of acrylonitrile, $138 \mathrm{meV}$, and the symmetry of both molecules is the same, $C_{s}$, making the dramatic increase of resonant width surprising.

With the large number of vibrational modes and the large resonance width, there is a quasi-continuum of resonances in the $0.19-1.0 \mathrm{eV}$ energy range. The spectra in Fig. 12 show a clear structure, however, indicating selectivity. Those resonances which lie on the diagonals $A, B$, and $C$ are clearly enhanced, as indicated by the corresponding labels in Fig. 12. Moreover, the excitation functions of the two inelastic peaks show enhancements at thresholds, more so than in acrylonitrile, indicative of stronger "unspecific" vibrational excitation at threshold for the larger molecule.

\section{F. Energy-loss spectra recorded along the diagonals}

The characteristics of vibrational excitation with respect to the various diagonals are revealed by the energy-loss spectra recorded at various constant residual energies, some of which are shown in Fig. 13. The center spectrum is recorded slightly below the diagonal $A$ and is, in view of the lifetime-imposed width of the resonance, indicative of both the diagonal $A$ and the slightly below-diagonal exceptions described above. The spectrum is dominated by the dominant excitations identified in the $2 \mathrm{D}$ spectrum, some of which, $v_{5}(\mathrm{C} \equiv \mathrm{N}$ str. $)$ and $v_{6}$ $(\mathrm{C}=\mathrm{C}$ str. $)$ and their overtones and combinations, are clearly related to the antibonding properties of the $\pi^{*}$ orbital, others $-v_{22}$ and $v_{11}$ - are not. The spectrum with $E_{r}=0.35 \mathrm{eV}$ was recorded on the diagonal $B$, where, interestingly, the "plausible" vibrations $v_{5}$ and $v_{6}$ are dominant. The bottom spectrum in Fig. 13 was recorded very near threshold. It shows little selectivity, all vibrations are excited with about the same intensity. The step around $\Delta E=0.15 \mathrm{eV}$ (i.e., at $E_{i}=E_{r}$ $+\Delta E=0.165 \mathrm{eV})$ is due to the onset of the $\pi^{*}$ resonance. (The onset is slightly below the nominal $A E_{a}=0.19 \mathrm{eV}$ because of the finite resonance width.) What causes vibrational excitation below this energy is uncertain, it could be hot bands, direct excitation by dipole mechanism, or dipole bound resonances.

The general trend that a near-continuum of vibrations, i.e., many overtones and combinations of low frequency modes, is excited near threshold and that this effect increases with increasing size of the molecule has been reported long ago for large molecules and has been termed "unspecific" excitation, i.e., excitation without selectivity for certain specific modes. Examples are given in Ref. 20 and in earlier references given therein. The theoretical description of the effect was presented by Gauyacq. ${ }^{21}$ The effect has recently been found to be 


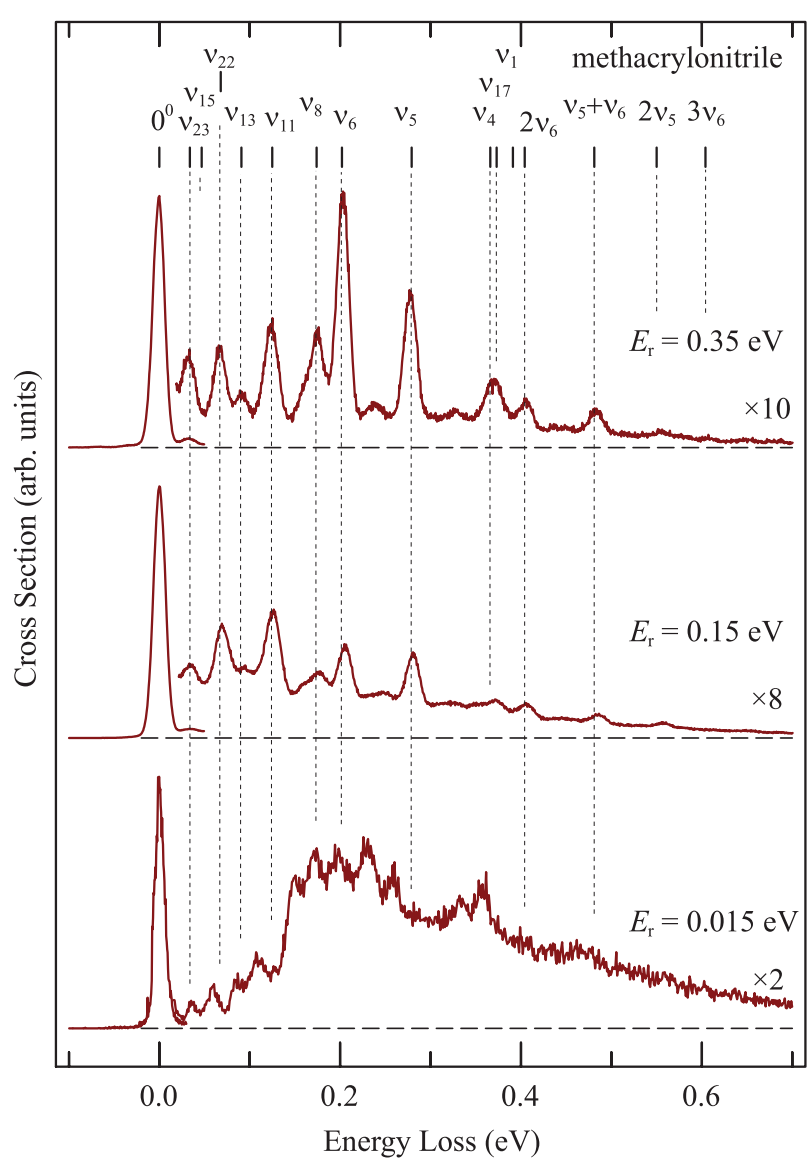

FIG. 13. Energy-loss spectra recorded with constant residual energies corresponding to various diagonals in the 2D spectrum of Fig. 11.

particularly pronounced in electron scattering from ionic liquids which thus behave as very large molecules in this sense. ${ }^{22}$

The excitation of low-frequency modes is related to intramolecular vibrational redistribution (IVR), whereby the incoming electron is initially coupled to a single mode as shown particularly clearly by the $2 \mathrm{D}$ spectra. Only few of the many modes of a polyatomic molecule couple strongly, determined by the initial slope of the anionic potential, given

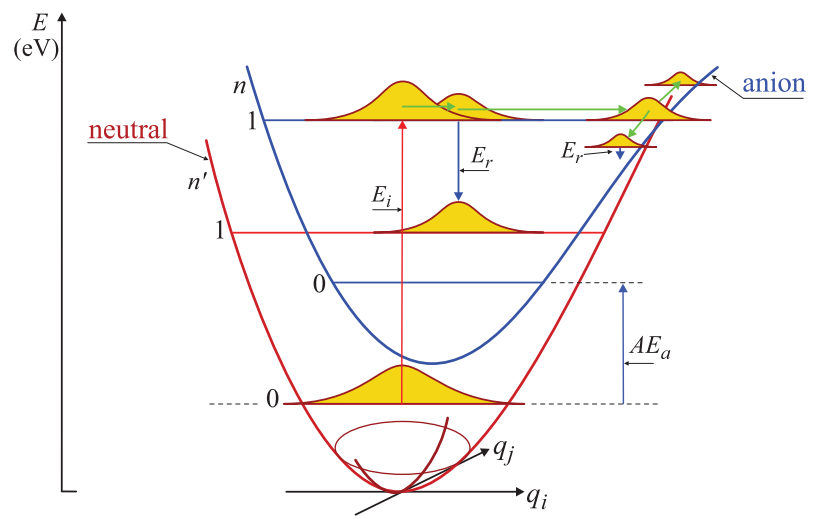

FIG. 14. Symbolic potential curves. Horizontally in the paper plane is the primarily excited mode $i$ like $\mathrm{C} \equiv \mathrm{N}$ stretch and perpendicularly to the paper plane is a representative low frequency mode $j$. Cross-anharmonicities convert the primarily excited mode $i$ into several low-frequency quanta along other modes. by the antibonding properties of the $\pi^{*}$ orbital. (Although in some of the present cases, the connection with the antibonding character of the $\pi^{*}$ orbital is not evident.) Exactly which of these few modes is selected is determined by the incident electron energy. The initial displacement of the nuclear wave packet occurs along this normal coordinate and an "early" detachment leads to excitation of this specific mode. If the nuclear wave packet survives to reach the outer turning point, then cross-anharmonicities may deviate it into other modes, leading to excitation of these other modes and detachment of a slow electron, as shown schematically in Fig. 14. The overall magnitude of the effect increases with the density of the "bath" modes, that is, with the number of normal modes and thus with the size of the molecule. This is also the essence of the theory of Gauyacq. ${ }^{21}$ This picture was also used by Fabrikant and coworkers to describe nondissociative electron attachment to $\mathrm{SF}_{6}{ }^{23,24}$

\section{CONCLUSIONS}

We show that 2D electron collision spectra contain rich information about the motion of a nuclear wave packet on a resonant potential surface - a key ingredient to understanding electron-induced chemistry. It is possible because the motion of the nuclear wave packet is characterized by a competition between propagation and detachment, the wave packet "rains down" along its path above the neutral potential surface and the final vibrational states carry (indirectly) information about where it rained down and thus allow us to "spy" on its trajectory.

Changing the incident electron energy allows us to choose in which initial vibrational state (or boomerang state when the autodetachment is fast and the state is lifetime broadened) the resonance is prepared, i.e., into what direction (along what normal mode) is the nuclear wave packet initially sent. The spectra of the detached electrons carry information about the packet propagation, they tell us into what mode and into which quanta the wave packet dropped, and whether it changed modes by IVR. The energy of the detached electron is indicative of the energy separation of the resonant and the neutral potential surfaces at the instant of detachment - detachment near the crossing seam of the two surfaces results in slow detached electrons.

We present 2D spectra for the lowest $\pi^{*}$ resonances of acrylonitrile and methacrylonitrile and report a number of observations.

- The width of the resonance in methacrylonitrile, about $100 \mathrm{meV}$, is much larger than in acrylonitrile, where it is about $15 \mathrm{meV}$. This finding is surprising in view of the small difference of the adiabatic attachment energies (190 meV and $138 \mathrm{meV}$, respectively) and in view of the fact that both compounds have the same symmetry.

- Prominent peaks are found along diagonals in the 2D spectra. The most prominent diagonal, labeled diagonal $A$, corresponds to $\Delta n_{j}=0$ processes where vibrational quanta $n$ do not change during detachment for all modes $j$. This indicates that if a wave packet is sent, by choosing the appropriate incident energy, into the direction of 
a certain normal mode $j$, then it "rains down" preferably along the same mode and without changing momentum, i.e., into a final state with the same number of quanta as the resonant state. (This argument becomes weakened for certain low frequency modes where the normal coordinates are significantly different for the neutral molecule and the anion.)

- Some of the prominent peaks correspond to modes expected on the grounds of the antibonding properties of the temporarily occupied orbital, in particular, the $\mathrm{C}=\mathrm{C}$ stretch and the $\mathrm{C} \equiv \mathrm{N}$ stretch modes. The reason for the prominence of other modes remains unexplained, particularly of the $\mathrm{C}-\mathrm{H}$ in-plane rocking mode $v_{7}$ and the in-plane $\mathrm{C}=\mathrm{C}-\mathrm{C}$ bending mode $v_{10}$ in acrylonitrile and the in-plane $\mathrm{CH}_{3}$ rocking mode $v_{11}$ and the $\mathrm{C}-\mathrm{C} \equiv \mathrm{N}$ out-of-plane bending mode $v_{22}$ in methacrylonitrile, because no particular driving force along these modes would be expected. It could be that the resonance width increases rapidly along these normal modes, so that a "hole" in the resonant surface is reached by a corresponding displacement, leading to rapid detachment.

- The less prominent diagonals, labeled $B$ and $C$, correspond to $\Delta n_{j}=-1$ processes where vibrational quanta of certain active modes decrease by one and vibrational energy is converted into electronic energy of the departing electron. The active modes are primarily the in-plane rocking mode $v_{7}$ and the $\mathrm{C} \equiv \mathrm{N}$ stretch mode $v_{4}$ in acrylonitrile. The large width of the resonances in methacrylonitrile makes the determination of the positions of the diagonals and the assignment of the detachment promoting modes less precise.

- Vibrational excitation between the diagonals, visualized by energy loss spectra recorded at the corresponding constant residual energies $E_{r}$, is weaker and less selective - many closely spaced peaks are observed.

- A special diagonal is the "threshold line," defined experimentally as an energy-loss spectrum recorded at a residual energy near zero. This excitation is more pronounced in methacrylonitrile than in acrylonitrile and the spectra consist of many closely spaced peaks of nearly the same intensity. These observations identify it as a manifestation of the "unspecific" vibrational excitation, well known in large molecules.

The picture emerging from these observations is one depicted in Fig. 14 where the nuclear wave packet is initially sent propagating in the direction of a normal mode defined by the energy of the incoming electron. Electrons detached in the initial phases of the relaxation result from selective excitation of specific modes and are positioned on the diagonals, primarily $A$, in the $2 \mathrm{D}$ spectra. The wave packet surviving this initial displacement arrives at portions of the potential surface where the anion and neutral surfaces approach or even cross, making detachment slow or impossible, where cross-anharmonicities make the motion switch modes in the sense of intramolecular vibrational redistribution. This leads to emission of generally slower electrons with low selectivity with respect to the final vibrational state. This picture is not new, of course, and has been invoked implicitly by the theory of unspecific vibrational excitation of Gauyacq ${ }^{21}$ and used in a model way by Fabrikant to describe nondissociative electron attachment to $\mathrm{SF}_{6} \cdot{ }^{23}$

We point out, however, that $2 \mathrm{D}$ spectra represent a powerful way of measuring and presenting the entire information in a concise way, which permits a number of insights already on a qualitative level of interpretation. Even more exciting, the 2D spectra open the possibility of a very detailed comparison between experiment and theory which would propagate a wave packet in a time dependent formalism in a way similar to that used by $\mathrm{Orel}^{3}$ to describe DEA in acetylene, except that both the VE and DEA would be included. The agreement in terms of the VE presented as a 2D spectrum would be a very insightful and sensitive test of the capacity of the theory to describe the correct trajectory of the wave packet on its way towards dissociation.

As an outlook, it will be interesting to record 2D spectra in a molecule where the DEA channel is also open and to include the DEA cross section in the 2D spectrum. DEA is situated beyond the crossing seam of the potential surfaces, that is, beyond the "threshold line" in the 2D spectrum.

\section{ACKNOWLEDGMENTS}

This research is part of Project No. 200020-144367/1 of the Swiss National Science Foundation and of the COST Action CM1301 CELINA.

${ }^{1}$ K. Regeta and M. Allan, Phys. Rev. Lett. 110, 203201 (2013).

${ }^{2}$ F. F. Gardner and G. Winnewisser, Astrophys. J. 195, 127 (1975).

${ }^{3}$ S. T. Chourou and A. E. Orel, Phys. Rev. A 80, 034701 (2009).

${ }^{4}$ O. May, J. Fedor, and M. Allan, Phys. Rev. A 80, 012706 (2009).

${ }^{5}$ M. Allan, J. Phys. B: At., Mol. Opt. Phys. 40, 3531 (2007).

${ }^{6}$ M. Allan, Phys. Rev. A 81, 042706 (2010).

${ }^{7}$ A. Gopalan, J. Bömmels, S. Götte, A. Landwehr, K. Franz, M. W. Ruf, H. Hotop, and K. Bartschat, Eur. Phys. J. D 22, 17 (2003).

${ }^{8}$ M. Allan, J. Phys. B: At., Mol. Opt. Phys. 38, 3655 (2005).

${ }^{9}$ J. C. Nickel, P. W. Zetner, G. Shen, and S. Trajmar, J. Phys. E: Sci. Instrum. 22, 730 (1989).

${ }^{10}$ R. K. Nesbet, Phys. Rev. A 20, 58 (1979).

${ }^{11}$ D. Chen and G. A. Gallup, J. Chem. Phys. 93, 8893 (1990).

${ }^{12}$ U. Varetto, Molekel 5.4, Swiss National Supercomputing Centre, Manno, Switzerland.

${ }^{13}$ J. M. Alía, H. G. M. Edwards, W. R. Fawcett, and T. G. Smagala, J. Phys. Chem. A 111, 793 (2007).

${ }^{14}$ J. Bragin, K. L. Kizer, and J. R. Durig, J. Mol. Spectrosc. 38, 289 (1971).

${ }^{15}$ P. D. Burrow, A. E. Howard, A. R. Johnston, and K. D. Jordan, J. Phys. Chem. 96, 7570 (1992)

${ }^{16}$ I. C. Walker, A. Stamatovic, and S. F. Wong, J. Chem. Phys. 69, 5532 (1978).

${ }^{17}$ M. Allan and L. Andrić, J. Chem. Phys. 105, 3559 (1996).

${ }^{18}$ R. Čurík, P. Čársky, and M. Allan, J. Chem. Phys. 142, 144312 (2015).

${ }^{19}$ C. Desfrançois, H. Abdoul-Carime, N. Khelifa, and J. P. Schermann, Phys. Rev. Lett. 73, 2436 (1994).

${ }^{20}$ M. Allan, J. Electron Spectrosc. Relat. Phenom. 48, 219 (1989).

${ }^{21}$ J. P. Gauyacq, J. Phys. B: At., Mol. Opt. Phys. 23, 3041 (1990).

${ }^{22}$ K. Regeta and M. Allan, "Free electrons and ionic liquids: Study of excited states by means of electron-energy loss spectroscopy and the density functional theory multireference configuration interaction method," Phys. Chem. Chem. Phys. (submitted).

${ }^{23}$ I. I. Fabrikant, Eur. Phys. J. D 35, 193 (2005).

${ }^{24}$ M. Braun, I. I. Fabrikant, M.-W. Ruf, and H. Hotop, J. Phys. B: At., Mol. Opt. Phys. 46, 195202 (2013). 